\title{
Effectiveness of Group Therapy Based on Acceptance and Commitment on the Severity of Fatigue and Depression With the Moderating role of Alexithymia in Patients With Multiple Sclerosis
}

\author{
Tahereh Haji Seyed Javadi $^{\circledR}$, Maryam Aghel Masjedi ${ }^{\circledR}{ }^{\circledR}$, Elnaz Hamzehloo ${ }^{3}{ }^{\circledR}$, Mir Javad Chehraghi $^{4}{ }^{\circledR}$, Leila

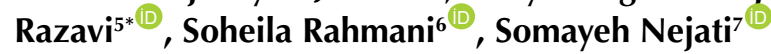

'Department of Psychology, Kish International Branch, Islamic Azad University, Kish Island, Iran ${ }^{2}$ Department of psychology, Rahman Institute of Higher Education, Ramsar, Mazandaran, Iran

${ }^{3}$ School of Psychology and Educational Sciences, Alameh Tabatabayi University of Tehran, Tehran, Iran

${ }^{4}$ Department of Psychology, Karaj Branch, Islamic Azad University, Karaj, Iran.

${ }^{5}$ School of Psychology and Educational Sciences, Islamic Azad University of Qom, Qom, Iran

${ }^{6}$ Department of Psychology, Karaj Branch, Islamic Azad University, Karaj, Iran

${ }^{7}$ School of Psychology and Educational Sciences, University of Semnan, Semnan, Iran

\begin{abstract}
Background: Alexithymia is a symptom that most psychosomatic patients experience and can affect their performance, psychological state, and the severity of their physical symptoms. Therefore, we aimed to determine the effectiveness of group therapy based on acceptance and commitment on the severity of fatigue and depression with the moderating role of alexithymia in patients with multiple sclerosis (MS) in Tehran.

Methods: In this research, a semi-experimental pre-test post-test design was used with three months of follow-up. Using convenience sampling, 40 patients with MS were randomly assigned to two experimental and control groups. Before and after the intervention and three months later in the follow-up phase, all participants responded to fatigue severity, depression, and alexithymia questionnaires. The intervention consisted of ten 90-minute group therapy sessions based on acceptance and commitment, which was performed only for the experimental group.

Results: Analysis of covariance indicated a significant difference between the mean scores of fatigue severity, depression, and alexithymia in the experimental and control groups in post-test and followup stages.

Conclusion: Group therapy based on acceptance and commitment with the moderating role of alexithymia effectively reduced the severity of fatigue and depression in patients with MS.

Keywords: Acceptance and commitment therapy; Severity of fatigue; Depression; Alexithymia; Multiple sclerosis.
\end{abstract}

*Correspondence to Leila Razavi,

MA in General Psychology, School of Psychology and Educational Sciences. Islamic Azad University of Qom, Qom, Iran.

Tel: +989126511300; Email: r.leila1355@gmail.com

Published online December 30,2020

Citation: Haji Seyed Javadi T, Aghel Masjedi M, Hamzehloo E, Chehraghi MJ, Razavi L, Rahmani S, Nejati S. Effectiveness of group therapy based on acceptance and commitment on the severity of fatigue and depression with the moderating role of alexithymia in patients With multiple sclerosis. Clin Neurosci J. 2021;8(1):37-43. doi:10.34172/icnj.2021.08.

\section{Introduction}

Multiple sclerosis (MS) is a neurological disease that leads to loss of myelin sheath in neurons. ${ }^{1}$ As a result, in different parts of the brain and spinal cord, the occurrence of the disease is accompanied by different symptoms in each patient, so that MS symptoms are not exactly identical in any two patients. ${ }^{2}$ Patients with MS face various problems, such as visual impairment, muscle weakness, coordination and balance problems, numbness in various organ, and cognitive problems. ${ }^{3}$ The occurrence rate of this disease is on the rise. Statistics show that more than 40000 people in Iran suffer from MS and about two thousand new cases are added to this group annually. ${ }^{4}$ Although the exact cause of MS is unknown, genetics, environmental factors, and infectious diseases appear to influence the emergence of the disease. It seems that MS causes mental issues such as depression, stress and anxiety, fatigue, and poor sleep quality, which can affect the severity of the disease and reduce the patients' quality of life. ${ }^{5}$ Fatigue is one of the symptoms of MS. It is an individual and internal symptom similar to pain yet distinct from muscular weakness. Fatigue is

(C) 2021 The Author(s). This is an open access article distributed under the terms of the Creative Commons Attribution License (http:// creativecommons.org/licenses/by/4.0/), which permits unrestricted use, distribution, and reproduction in any medium, provided the original work is properly cited. 
an unpleasant mental feeling that deteriorates the physical and mental performance of the individual and prevents the patients from doing their daily tasks. This symptom is also a major contributor to social isolation, depression, unemployment, limitations in performing daily activities, and reduction in the quality of life in these patients. ${ }^{6,7}$ Studies show that fatigue in patients with MS is associated with higher inability and lower quality of life. ${ }^{8}$ According to some studies, fatigue management in these patients has had a positive effect not only on the severity of fatigue but also on the reduction of their depression symptoms. ${ }^{9}$ After being diagnosed with MS, patients experience severe stress and think they can no longer manage their social and occupational roles and cannot interact with others as a healthy person. Therefore, depression and anxiety are common in such patients. ${ }^{10}$ Depression in patients with MS increases the incidence of cognitive errors, suicide, relationship problems, and reduces adaptation to the disease $^{11}$ and rate of daily activities, and leads to lack of motivation, and ultimately lack of physical and mental health and dissatisfaction with life. ${ }^{12}$ Alexithymia is one of the most common problems in people with MS. It seems that these patients have numerous problems with cognitive processing of emotional information and emotional regulation. ${ }^{13}$

People with alexithymia, defective cognitive processes, and limited emotional awareness struggle with persistent physiological excitation and neural reactions, resulting in damage to their autoimmune system, pituitary-adrenal axis, and endocrine system. ${ }^{14}$ Moreover, alexithymia is accompanied by self-destructive behaviors such as drug and alcohol abuse, eating disorders, maladaptive behaviors, as well as pain intensity and increased symptoms of physical illnesses. ${ }^{15}$ Alexithymia is also associated with physical disorders such as $\mathrm{MS}^{16}$ and mental disorders such as depression. ${ }^{17}$ It seems that alexithymia, as a moderating factor, affects the severity of fatigue and depression in patients with MS. In one study, ${ }^{18}$ the difficulty in identifying and describing emotions as two components of alexithymia in patients with asthma predicted depression and anxiety. Rostami Nezhad and colleagues $^{19}$ found a significant relationship between alexithymia and depression, stress, anxiety, and fatigue in people undergoing addiction treatment. Various drugs and non-drug interventions have been proposed for the treatment of MS-related depression and fatigue. Since anti-fatigue and depression drugs often have side effects, it is recommended that non-pharmacological treatments be used to reduce the risk of such symptoms. ${ }^{20}$

Acceptance and commitment therapy is a nonpharmacological treatment that teaches the patient to view thoughts and emotions (such as sorrow or pain) as dynamic events that are not problematic per se; rather, the response to them (such as avoidance) can lead to problems in life. ${ }^{21}$ Unlike many therapies that emphasize the reduction or control of symptoms, acceptance and commitment therapy emphasizes the acceptance of negative reactions (such as thoughts and negative emotions).22 According to this model, people learn to accept their thoughts and emotions in the first place, to live in the present, and to be more flexible. This treatment tries to increase a person's ability to stay active and act in accordance with personal values in order to function better and have a higher quality of life. ${ }^{23}$ Several studies have shown the positive effect of acceptance and commitment therapy on improving the quality of life and performance of people with chronic diseases. ${ }^{24,25}$ Therefore, this treatment can also be used to help patients with MS. Baseri and Bozorgi ${ }^{22}$ showed that group acceptance and commitment therapy significantly increased cognitive emotion regulation and reduced alexithymia in patients with type 2 diabetes mellitus. Another study indicated that acceptance and commitment therapy can be used to significantly reduce fatigue and anxiety in patients with breast cancer. ${ }^{25}$ Considering the significant role of psychological factors in developing psychosomatic disorders, such studies are important for minimizing the occurrence of these conditions or improving the living conditions of patients with MS. Therefore, we aimed to assess the effectiveness of group therapy based on acceptance and commitment on the severity of fatigue and depression with the moderating role of alexithymia in patients with MS.

\section{Materials and Methods}

This study employs a semi-experimental pre- and posttest design and a control group with a follow-up period of three months. The target population includes all patients with MS who referred to the MS Society in Tehran during spring 2018. The sampling process started by selecting 100 patients with MS from the list provided by the Society. Then these patients were invited via SMS to participate in the study. From among the people willing to participate in the research project, screening was done and 40 subjects were selected through purposive sampling. After obtaining their informed consent, They were then randomly assigned to two groups, 20 patients in the experimental group (undergoing acceptance and commitment treatment) and 20 participants in the control group. The inclusion criteria were: diagnosis of MS by a neurologist, the absence of acute or chronic disorders, not using psychiatric drugs during the study, minimum education level of high school diploma in patients aged 20-50 years with a history of 5-10 years of illness. The exclusion criteria were unwillingness to cooperate in treatment sessions and being absent for more than two sessions. Participants in the experimental and control groups completed the questionnaires at three times, before the intervention (pre-test), after the intervention (post-test), and three months after the intervention (follow-up). The experimental group received therapy 
by participating in ten 90 -minute sessions. The control group did not receive any treatment interventions. Due to ethical considerations, at the end of the study, the treatment was also provided for the control group.

\section{Fatigue Severity Scale}

Fatigue Severity Scale (FSS) has been developed by Krupp et al, for assessing the severity of fatigue in chronic diseases such as MS and lupus. This scale measures fatigue in 9 items based on 7-point Likert scale and higher scores indicate greater fatigue. Cronbach's alpha of this scale for healthy people, MS patients, and lupus patients has been reported to be $0.88,0.81$, and 0.89 , respectively. ${ }^{26}$ The internal consistency (Cronbach's alpha) and test-retest reliability of the Farsi version of this scale have been obtained equal to 0.98 and 0.93 , respectively. ${ }^{26}$ In the present study, test-retest reliability (within two months) and internal consistency (Cronbach's alpha) of FSS were calculated 0.86 and 0.89 , respectively.

\section{Beck Depression Inventory-II}

The original Beck Depression Inventory (BDI) was first created in 1961 by Beck and colleagues. ${ }^{27}$ The BDI II consists of 21 items. The general purpose of this study is to measure the severity of depression (from nondepressed to severe) among the participants. Respondents use a four-point Likert scale from zero to three $(0=$ never, $1=$ seldom, 2 = sometimes, $3=$ often) to evaluate their experience during the previous week. Depression score was calculated by adding scores for related items. ${ }^{28}$ In a study with a normal sample, the internal consistency for the depression scale was as high as 0.84 . In a clinical study, the internal consistency coefficient was $0.96 .^{29}$

In a non-clinical study, the internal consistency coefficient (Cronbach's alpha) of the depression scale was 0.93 . These coefficients are all greater than the 0.70 threshold recommended by Nunnally and Bernstein. ${ }^{30}$ In this study, its reliability was calculated using the retest method $(\mathrm{r}=$ 0.92).

\section{Toronto Alexithymia Scale (TAS-20)}

This 20 item scale was developed by Bagby and coworkers in 1994. In addition to the overall score, it also evaluates alexithymia in the three dimensions of difficulty identifying feelings (seven items), difficulty describing feelings (five items), and externally oriented thinking (eight items). In this scale, higher scores indicate the greater severity of alexithymia. ${ }^{31}$ The reliability of this scale was 0.98 for an Iranian sample for all three dimensions. The validity of this scale was reported to be $0.87 .{ }^{32}$ In this study, its reliability was $r=0.81$ using the retest method. The session protocol for acceptance based commitment treatment is presented in Table 1.

\section{Results}

The collected data were analyzed using mean, standard deviation (SD), frequency distribution tables, and onevariable analysis of covariance test using SPSS software, version. In this study, 40 patients with MS aged 20-50 years (20 patients in the experimental group and 20 in the control group) were enrolled.

As it is shown in Table 2, the mean \pm SD age of the participants' in the control and experimental group were $24.67 \pm 3.72$ and $25.86 \pm 4.18$ years, respectively.

As shown in Table 3, equal variances assumption was confirmed for all of the components. The MANCOVA results indicated that the treatment was effective ( $P$ $<0.001, F=31.334$, Pillai's trace $=0.976$ ). In order to study patterns of difference, one-variable analysis of covariance was used.

As shown in Table 4, there has been persistence in the components that have been effective in the treatment.

The results in Table 5 confirm the moderating role of alexithymia in the effectiveness of acceptance and commitment based therapy on depression and fatigue in patients with MS.

\section{Discussion}

The aim of this study was to determine the effectiveness of group therapy based on acceptance and commitment with the moderating role of alexithymia on the severity of fatigue and depression in patients with MS. The results have been consistent not only in the post-test but also in the three-month follow-up phase. The findings showed that group therapy based on acceptance and commitment had a significant role in decreasing the severity of fatigue and symptoms of depression by improving alexithymia. To the best of the authors' knowledge, no similar research has been done on this population of patients with MS, yet these results are in overall agreement with the findings reported by Darvish Baseri and Rajabi et $\mathrm{al}^{33}$ as well as Salehi et $\mathrm{al}^{34-35}$. Salehi and colleagues showed that acceptance and commitment based therapy can reduce the psychological problems (such as stress, anxiety, and depression) of patients with fibromyalgia syndrome by creating and developing acceptance and increasing valuebased action, which gives patients a better life in spite of their pain and illness. ${ }^{36}$ Parsa et al ${ }^{37}$ showed that the acceptance and commitment group therapy positively influenced health, burnout, pain, mental health, and perception of issues, but had no effect on supporting areas and sexual satisfaction. In general, this treatment has increased the quality of life in patients with MS. Tilaki et $\mathrm{al}^{38}$ showed that acceptance and commitment training had positive effects on psychological flexibility and alexithymia in women with chronic pain.

In explaining the effect of group therapy based on acceptance and commitment on reducing the severity of fatigue with the moderating role of reduced alexithymia, it can be argued that most patients with MS experience fatigue and view this problem as the worst symptom of their illness which has adverse effects on daily activity, 
Table 1. Summary of the Instructions for the Acceptance and Commitment Based Therapy Sessions

Introducing team members and performing pre-test, discussing the behavior limits and explaining the rules and principles governing the counseling sessions, the informed consent of the group members for the treatment process, presentation of the objectives and explaining the possibility for change. Explaining the philosophy of intervention based on this approach, introduction to creative hopelessness: in this context, it is possible to present content about ignoring the values, isolation, reactions, and avoidance (explaining the concepts of fatigue severity and depression) and introducing inefficient systems of the past and alexithymia (familiarity with previous efforts to accomplish objectives).

Introduction to acceptance and commitment therapy concepts (psychological flexibility, psychological acceptance, psychological awareness, cognitive defusion, self as context, personal narrative, value clarification, and committed action) in six steps as follows:

(1) In this treatment, first, the psychological acceptance of mental experiences (thoughts, feelings, etc.) is increased and ineffective attempts to control them are reduced. The patient is taught that any action to avoid or control these unwanted mental experiences is ineffective and may exacerbate them. These experiences must be fully accepted without any internal or external reaction to remove them; (2) the psychological $2^{\text {nd }}$ session awareness of the individual about the present moment is increased, in other words, people become aware of their mental states, thoughts, and behavior at the moment; (3) the patient is taught to separate himself/herself from these mental experiences (cognitive defusion) to be able to function independently of these experiences; (4) trying to reduce the excessive focus on self-image or the personal narrative that a person has for himself in his mind; 5) helping people to identify and clearly state their personal values and transform them into specific behavioral objectives (clarification of the values); and (6) motivating them to act with commitment; that is acting toward their identified objectives and values while accepting their mental experiences.

Reviewing the previous session's assignments: People's response to control strategies, control as a form (control is a problem, not a solution)

$3^{\text {rd }}$ session (teaching that any action to avoid or control unwanted mental experiences is ineffective), assignments: taking notes of daily experiences, daily notes about inclinations, daily notes about clean and dirty discomfort and moving toward creative hopelessness.

$4^{\text {th }}$ session Performance evaluation; Examining individual experiences since the previous session; Assessing assignments. Assignments: Mindfulness Exercise, what is mindfulness, impact on identification and awareness, and beginning exercise on clarification of values.

$5^{\text {th }}$ session Performance evaluation; Session objective: distinguishing self-conceptualization from self-observation, assess the patient's ability to defuse from thoughts and feelings, continue mindfulness experience and value clarification.

The goal of this session is to show the importance of values and to inform people how values and understanding them makes "inclination /

$6^{\text {th }}$ session acceptance" to appear valuable, values as behaviors versus values as feelings, exercising mindfulness about bodily feelings and discussing them, exercises about positive fatigue

The goal of this session is to help people in the group to recognize the severity of fatigue which is not in accordance with their values. People must continuously understand their values as a source of commitment and apply them toward the implementation of the concept (inclination) and in the service of behavioral activation and personal objectives. Options against judgments / decisions, identifying value-based behaviors must be reviewed within a week. Identification of values (smaller goals in the service of greater ones). Losing weight to reduce fatigue.

The goal of this session is to discover the relationship between objectives and activities, and to strengthen the factors of inclination and defusion, to help achieve behavioral objectives. Also, the assignments should be continued toward the behavioral activation feature in the form of commitment to specific activities which are determined based on greater objectives and values identified by the participants. Continuing to set effective goals related to values, continuing mindfulness exercises even when walking, discussing fatigue level and alexithymia.

$9^{\text {th }}$ session Teaching mindfulness (emotional awareness and wise mindfulness), teaching about the skills that are observed and described, and how skills are not judged, staying focused and learning how such skills work.

Evaluation of committed actions; Training the participants to be therapists themselves, to maximize the possibility of them expressing the skills learned during the treatment and using these skills after the completion of the intervention. Preparation to deal with possible failures after the end of the treatment.

Identifying possible strategies, barriers of the FEAR algorithm, committed action based on the acceptance and commitment therapy algorithm, and the main message of acceptance and commitment therapy. Providing a summary of the previous sessions and administering the post-test. Suggested assignments: Registering participant objectives, for example, one to six months of discussion and modification of the program after the treatment, and relapse prevention: Sessions can be continued on a monthly basis if requested by the participants, as well as 30 minutes or shorter sessions by telephone.

Table 2. Descriptive Statistics of the Mean and Standard Deviation of the Age Variable

\begin{tabular}{lccccc}
\hline Group & Number & Mean & Standard Deviation & Min & Max \\
\hline Control & 20 & 24.67 & 3.72 & 20 & 41 \\
Experimental & 20 & 25.86 & 4.18 & 21 & 42 \\
\hline
\end{tabular}

Table 3. Testing the Assumption of Equal Variances for the Research Variables

\begin{tabular}{lcccc}
\hline Variable & $\mathbf{F}$ & df1 & df2 & P Value \\
\hline Depression & 2.109 & 1 & 22 & 0.161 \\
Fatigue Severity & 1.644 & 1 & 22 & 0.213 \\
Alexithymia & 15.243 & 1 & 22 & 0.001 \\
\hline
\end{tabular}

Table 4. Analysis of Variance for Variables of the Experimental Group in the Post-test Phase

\begin{tabular}{lcccccc}
\hline Source of Variations & $\begin{array}{c}\text { Sum of } \\
\text { Squares }\end{array}$ & $\mathbf{d f}$ & $\begin{array}{c}\text { Mean } \\
\text { Square }\end{array}$ & $\mathbf{F}$ & $\boldsymbol{P}$ Value & $\boldsymbol{\eta}^{2}$ \\
\hline Depression & 70.042 & 1 & 70.042 & 48.279 & 0.001 & 0.687 \\
Fatigue Severity & 2.042 & 1 & 2.042 & 1.905 & 0.181 & - \\
Alexithymia & 10.667 & 1 & 10.667 & 7.866 & 0.010 & 0.263 \\
\hline
\end{tabular}

Table 5. Investigation of the Moderating Role of Alexithymia in Depression and Fatigue

\begin{tabular}{lccccccc}
\hline Source of Variations & $\begin{array}{c}\text { Sum of } \\
\text { Squares }\end{array}$ & df & $\begin{array}{c}\text { Mean } \\
\text { Square }\end{array}$ & $\mathbf{F}$ & $\begin{array}{c}\boldsymbol{P} \\
\text { Value }\end{array}$ & $\boldsymbol{\eta}^{2}$ \\
\hline Alexithymia & 1190.042 & 1 & 1190.042 & 41.170 & 0.001 & 0.652 \\
\hline$P \leq 0.001$. & & & & & &
\end{tabular}


functioning, and quality of life in patients with MS. In fact, the intervention of acceptance and commitment, instead of suppressing and avoiding feelings, empowers the patients with MS to accept negative experiences and emotions and lead a meaningful and valuable life. By improving psychological acceptance and increasing meaningful and flexible activities, this treatment enhances the patient's ability to perform effectively in line with individual values, despite pain and suffering (psychological flexibility). ${ }^{39}$ After a while, this new perspective of values and goals of life creates positive behavioral changes, leading to a more meaningful life and ultimately a reduction in negative emotions and depression. In fact, the purpose of this treatment is not to increase realistic and rational thinking with the encouragement of emotions, rather the goal of this therapeutic approach is to reduce the avoidance of psychological experiences and increase awareness of them to shift the focus on the present moment. ${ }^{35}$ This treatment seems to teach patients not to identify themselves with the thoughts and feelings caused by their illness. As a result, people are released from the downward spiral of negative thoughts and emotions (anxiety, stress, despair, hopelessness, and depression). This ultimately increases emotional awareness and cognitive emotion regulation. ${ }^{36}$ This will, in turn, lead to better social, physical, and emotional performance, ${ }^{25}$ resulting in reduced fatigue in patients.

In explaining the effect of acceptance and commitment therapy on reducing depression and the role of alexithymia, one can often admit that symptoms of depression are among the most common problems patients with MS encounter. In fact, one source of their depression is the inability to overcome past experiences and related events. By participating in acceptance and commitment group therapy, the patient actually learns to accept all the agonizing and negative feelings of the past and does not attempt to control them. It also helps them to identify crucial aspects of life and use their values to guide behavioral changes in life. Therefore, using acceptance techniques, people believe that they are able to cope with the challenges in their personal, family, and social life. This treatment enables people to get in touch with the present moment and accept their mental experiences and body feelings. As a result, this treatment positively affects emotion regulation and gives more meaning to life while reducing mental ruminations associated with the stressors. As a consequence, alexithymia and depression symptoms are reduced as well. ${ }^{34,37}$

Therefore, it seems that the reason for the effectiveness of group therapy based on acceptance and commitment with the moderating role of alexithymia in patients with MS in reducing the severity of fatigue and depression is the result of a change in their attitudes toward irrational thoughts and the negative cycle created by these thoughts. In fact, this treatment provides therapeutic changes through the development of awareness, acceptance, cognitive defusion, and value-oriented action, leading to psychological flexibility in patients. Ultimately, increased psychological flexibility can lead to identifying, describing, and expressing emotions that will improve the psychological conditions of patients with MS.

Finally, it should be noted that this research has been subject to some limitations. Convenience sampling and lack of control over some intervening variables are among these limitations. Therefore, caution should be exercised in generalizing the results.

\section{Conclusion}

Group therapy based on acceptance and commitment with the moderating role of alexithymia has been effective in improving sleep quality, fatigue severity, and depression in the post-test and follow-up stages in patients with MS. Therefore, this therapeutic approach is recommended as a means to reduce the psychological symptoms in these patients. For future studies, it is recommended to investigate the effectiveness of this treatment for other symptoms of MS as well as other chronic diseases.

\section{Conflict of Interest}

The authors declare that they have no conflict of interests.

\section{Acknowledgments}

The authors greatly appreciate the cooperation of staff and members of the "Multiple Sclerosis Society of Tehran" whose participation made it possible to conduct this research.

\section{Funding/Support}

There was no funding or support.

\section{Ethical Statement}

Not applicable.

\section{References}

1. Wingerchuk DM, Carter JL. Multiple sclerosis: current and emerging disease-modifying therapies and treatment strategies. Mayo Clin Proc. 2014;89(2):225-40. doi: 10.1016/j. mayocp.2013.11.002.

2. Azimian M, Shahvarughi-Farahani A, Rahgozar M, Etemadifar $M$, Nasr Z. Fatigue, depression, and physical impairment in multiple sclerosis. Iran J Neurol. 2014;13(2):105-7.

3. Motaharinezhad F, Seyed S, Zeinali R. The impact of mental practice on sleep quality, fatigue, functional balance and gaiting in patient with multiple sclerosis: A case report. Koomesh. 2016;17(4):944-9. [Persian].

4. Albuquerque C, Geraldo A, Martins R, Ribeiro O. Quality of life of people with multiple sclerosis: clinical and psychosocial determinants. Procedia Soc Behav Sci. 2015;171:359-65. doi: 10.1016/j.sbspro.2015.01.133.

5. Čarnická Z, Kollár B, Šiarnik P, Krížová L, Klobučníková K, Turčáni P. Sleep disorders in patients with multiple sclerosis. J Clin Sleep Med. 2015;11(5):553-7. doi: 10.5664/jcsm.4702.

6. Motaharinezhad F, Parvaneh S, Ghahari S. Fatigue in People with Multiple Sclerosis: Cause, Evaluation and Treatment. Journal of Paramedical Sciences \& Rehabilitation. 
2016;5(1):73-80. doi: 10.22038/jpsr.2016.6386. [Persian].

7. Veauthier C, Hasselmann H, Gold SM, Paul F. The Berlin Treatment Algorithm: recommendations for tailored innovative therapeutic strategies for multiple sclerosis-related fatigue. EPMA J. 2016;7(1):25. doi: 10.1186/s13167-016-0073-3.

8. Salehpoor G, Kafi SM, Rezaei S, Hosseininezhad M, Salehi I. The relation between fatigue severity with psychological symptoms and quality of life in multiple sclerosis. Zahedan Journal of Research in Medical Sciences. 2012;14(9):80-6. [Persian].

9. Pagnini F, Bosma CM, Phillips D, Langer E. Symptom changes in multiple sclerosis following psychological interventions: a systematic review. BMC Neurol. 2014;14:222. doi: 10.1186/ s12883-014-0222-z.

10. Simpson R, Booth J, Lawrence M, Byrne S, Mair F, Mercer $\mathrm{S}$. Mindfulness based interventions in multiple sclerosis-a systematic review. BMC Neurol. 2014;14:15. doi: 10.1186/1471-2377-14-15.

11. Moore P, Hirst C, Harding KE, Clarkson H, Pickersgill TP, Robertson NP. Multiple sclerosis relapses and depression. J Psychosom Res. 2012;73(4):272-6. doi: 10.1016/j. jpsychores.2012.08.004.

12. Dehghani A, Mohamad Khan Kermanshahi S, Memarian R. Abundance of stress, anxiety and depression in multiple sclerosis patients. Alborz University Medical Journal. 2013;2(2):82-8. doi: 10.18869/acadpub.aums.2.2.82. [Persian].

13. Chalah MA, Ayache SS. Alexithymia in multiple sclerosis: a systematic review of literature. Neuropsychologia. 2017;104:31-47. doi: 10.1016/j. neuropsychologia.2017.07.034.

14. Kano M, Fukudo S. The alexithymic brain: the neural pathways linking alexithymia to physical disorders. Biopsychosoc Med. 2013;7(1):1. doi: 10.1186/1751-0759-7-1.

15. Cerutti R, Calabrese $M$, Valastro $C$. Alexithymia and personality disorders in the adolescent non-suicidal self injury: preliminary results. Procedia Soc Behav Sci. 2014;114:372-6. doi: 10.1016/j.sbspro.2013.12.714.

16. Eboni ACB, Cardoso M, Dias FM, da Gama PD, Gomes $\mathrm{S}$, Goncalves MVM, et al. High levels of alexithymia in patients with multiple sclerosis. Dement Neuropsychol. 2018;12(2):212-5. doi: 10.1590/1980-57642018dn12020015.

17. Rutten EA, Bachrach N, van Balkom AJ, Braeken J, Ouwens MA, Bekker MH. Anxiety, depression and autonomyconnectedness: the mediating role of alexithymia and assertiveness. Psychol Psychother. 2016;89(4):385-401. doi: 10.1111/papt.12083.

18. Rezaei F, Karimi F, Afshari A, Hosseini-Ramaghani N. The correlation between alexithymia and anxiety, depression in asthma. Feyz. 2017;21(2):178-87. [Persian].

19. Rostami Nezhad S, Mazloumi Rad M, Farrokhi N, Viesy F, Ghahari S. The relationship of alexithymia with depression, anxiety, stress, and fatigue among people under addiction treatment. Ann. Trop. Med. Public Health. 2017;10(6):1698703. doi: 10.4103/atmph.atmph_589_17.

20. Hulbert-Williams NJ, Storey L, Wilson KG. Psychological interventions for patients with cancer: psychological flexibility and the potential utility of Acceptance and Commitment Therapy. Eur J Cancer Care (Engl). 2015;24(1):15-27. doi: 10.1111/ecc.12223.

21. Burckhardt R, Manicavasagar V, Batterham PJ, Hadzi-Pavlovic
D, Shand F. Acceptance and commitment therapy universal prevention program for adolescents: a feasibility study. Child Adolesc Psychiatry Ment Health. 2017;11:27. doi: 10.1186/ s13034-017-0164-5.

22. Darvish Baseri L, DashtBozorgi Z. Effectiveness of Group Therapy Based on Acceptance and Commitment on Cognitive Emotion Regulation and Alexithymia of Patients with Type 2 Diabetes. IJPN. 2017; 5 (1) :7-14.

23. Nourian L, Aghaei A, Ghorbani M. The efficacy of acceptance and commitment therapy on weight self-efficacy lifestyle in obese women. Journal of Mazandaran University of Medical Sciences. 2015;25(123):159-69. [Persian].

24. Ghalian Nozari F, Hassanzadeh R, Gholamnejad S. Effectiveness of acceptance and commitment therapy on ineffective sexual attitudes and sexual function in pregnant women. medical journal of mashhad university of medical sciences, 2020; 62(5.1): 624-631. doi: 10.22038/ mjms.2020.15525.

25. Mousavi M, Hatami M, Nouryghasemabadi R. The evaluation of effect of acceptance and commitment therapy on fatigue and anxiety in patients with breast cancer. Research in Medicine. 2017;41(3):189-98. [Persian].

26. Shahvaroughi Farahani A, Azimiyan $M$, Fallahpour $M$, Karimlou M. Fatigue Severity Scale (FSS): evaluation of reliability of the Persian version among persons with multiple sclerosis. Archives of Rehabilitation. 2010;10(4). [Persian].

27. Wan Mahmud WM, Awang A, Herman I, Mohamed MN. Analysis of the psychometric properties of the malay version of Beck Depression Inventory II (BDI-II) among postpartum women in Kedah, North West of Peninsular Malaysia. Malays J Med Sci. 2004;11(2):19-25.

28. Gallagher D, Nies G, Thompson LW. Reliability of the Beck Depression Inventory with older adults. J Consult Clin Psychol. 1982;50(1):152-3. doi: 10.1037//0022-006x.50.1.152.

29. Ghassemzadeh H, Mojtabai R, Karamghadiri N, Ebrahimkhani N. Psychometric properties of a Persian-language version of the Beck Depression Inventory--Second edition: BDI-IIPERSIAN. Depress Anxiety. 2005;21(4):185-92. doi: 10.1002/ da. 20070 .

30. Steer RA, Ball R, Ranieri WF, Beck AT. Dimensions of the Beck Depression Inventory-II in clinically depressed outpatients. J Clin Psychol. 1999;55(1):117-28. doi: 10.1002/(sici)10974679(199901)55:1<117::aid-jclp12>3.0.co;2-a.

31. Leising D, Grande T, Faber R. The Toronto Alexithymia Scale (TAS-20): a measure of general psychological distress. J Res Pers. 2009;43(4):707-10. doi: 10.1016/j.jrp.2009.03.009.

32. Stivaleti Colombarolli M, Carolina Zuanazzi A, Koich Miguel F, Giromini L. Psychometric properties of the Toronto Alexithymia Scale (TAS-20) in Brazil. Transcult Psychiatry. 2019;56(5):992-1010. doi: 10.1177/1363461519847312.

33. Rajabi S, Yazdkhasti F. The effectiveness of acceptance and commitment group therapy on anxiety and depression in women with MS who were referred to the MS association. Journal of clinical psychology. 2014 Mar 21;6(1):29-38. [Persian].

34. Salehi N, Neshat Doost HT, Afshar HT. The Impact of Group Therapy Based on Acceptance and Commitment on Psychological Indicators (Depression, Anxiety, and Stress) in Women with Fibromyalgia. RBS. 2018; 16 (1) :78-83. [Persian].

35. Wynne B, McHugh L, Gao W, et al. Acceptance and Commitment Therapy Reduces Psychological Stress in Patients 
With Inflammatory Bowel Diseases. Gastroenterology. 2019;156(4):935-945.e1. doi: 10.1053/j.gastro.2018.11.030.

36. Salehi N, Taher Neshat Dust H, Afshar H, Effectiveness of group therapy based on acceptance and commitment on psychological measures (depression, anxiety, and stress) in female fibromyalgia patients. Journal of Behavioral Sciences. 2018;16(1):78-83.

37. Parsa M, Sabahi P, Mohammadifar MA. The effectiveness of acceptance and commitment group therapy to improving the quality of life in patients with multiple sclerosis. J Clin Psychol. 2018;10(1):21-8. doi: 10.22075/jcp.2018.11686.1156.
[Persian].

38. Tilaki M, Taher M, Mojarrad A, Jafari Sani B. Efficacy of acceptance and commitment therapy on psychological flexibility and alexithymia of women with chronic pain. Psychol Stud. 2018;14(3):147-64. [Persian].

39. Golshani G, Pirnia B. Comparison of Mindfulness-Based Cognitive Therapy (MBCT) with Acceptance and Commitment Therapy (ACT) on the severity of fatigue, improvement of sleep quality and resilience in a patient with prostate cancer: a single-case experimental study. Int J Cancer Manag. 2019;12(2):e88416. doi: 10.5812/ijcm.88416. 